\title{
Reconstructing Crossing Muons in ICARUS Detector
}

Michael Vayninger, Mentor: Bruce Howard

Poster\#FERMILAB-POSTER-21-084-STUDENT

\section{Objective}

The ICARUS detector's dual cryostat geometry, high beam energy, and $6^{\circ}$ angle to the NuMI beam line allows for tracks from neutrino interactions (usually muons) to cross from one cryostat to the other, which is more common for higher energy events.

- By reassembling crossing tracks and using the total track length, it is possible to make an accurate momentum estimate, which is useful for many studies

- It may also be possible to recover more events for use in analysis by loosening restrictions on events with crossing tracks by allowing them to not be within the fiducial volume (vertex far from the edges of the cryostats)
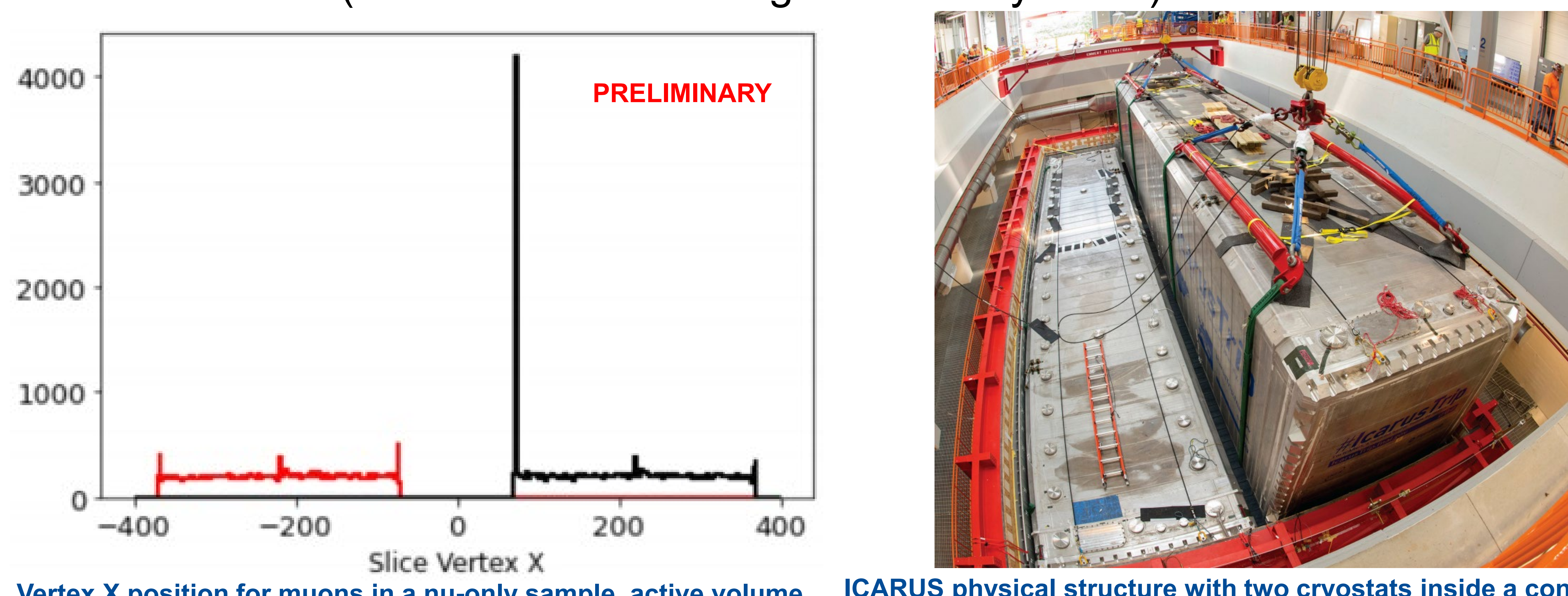
Vertex $X$ position for muons in a nu-only sample, active volume ICARUS physical structure with two cryostats inside a containmen
edges are at $X= \pm 369, \pm 72 \mathrm{~cm}$. The spike at $X=72$ is due to crossing chamber, each filled with liquid argon (LAr) and housing detector track.
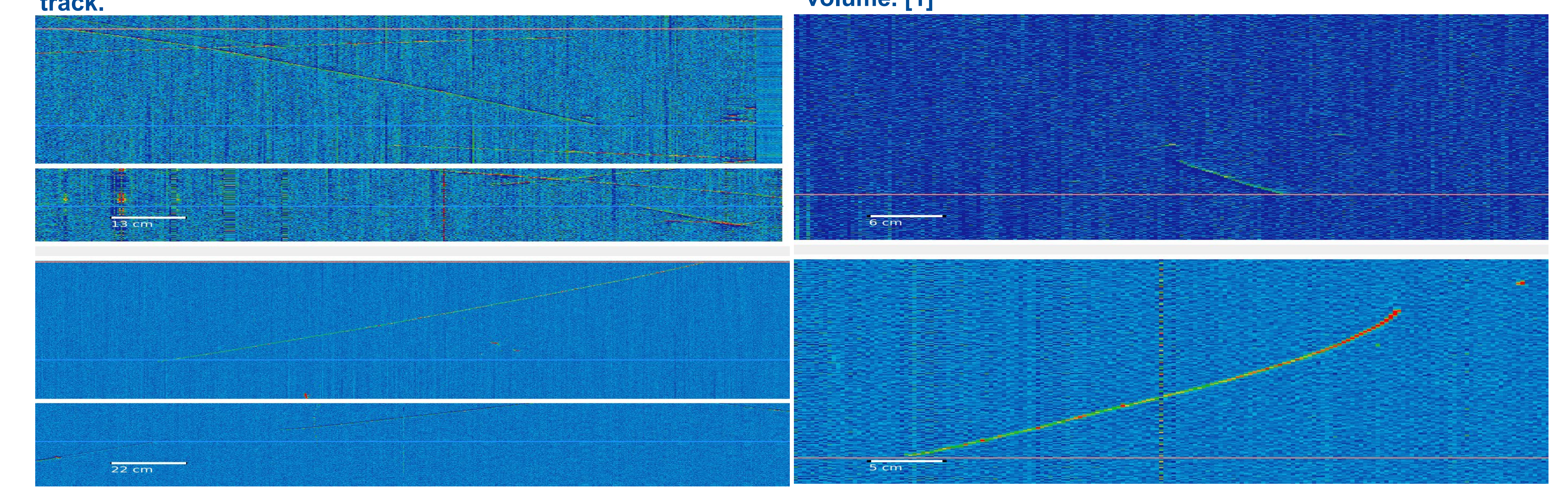

Crossing track candidate in data, vertex (bottom of left image) creates a track that passes through cryo0 and appears at the cryo1 anod

Acknowledgment: I would like to thank all those who work as part of SBN and ICARUS for their work which enabled this study, the SULI internship for their organization, and my mentor Bruce Howard for his help in conducting the entire study.

[1] Hahn, Reidar. 2018. Available at Fermilab VMS 18-0150-27 [Accessed August 3, 2021]

This manuscript has been authored by Fermi Research Alliance, LLC under Contract No. DE-AC02-07CH11359 with the U.S. Department of Energy, Office of Science, Office of High Energy Physics.

\section{Finding Crossing Candidates}

To find crossing candidates, a series of cuts are applied to attempt to isolate signal events, which we define as crossing tracks from a nu event:

- Cut tracks that are tagged by the reconstruction chain as being very likely cosmic, slices that are determined to be very cosmic-like, and tracks with a poor match between its scintillation light and TPC charge

- Tag tracks with endpoints near the $x= \pm 72 \mathrm{~cm}$ anodes and pair them up as candidates

- Cut on deviation and change in track direction

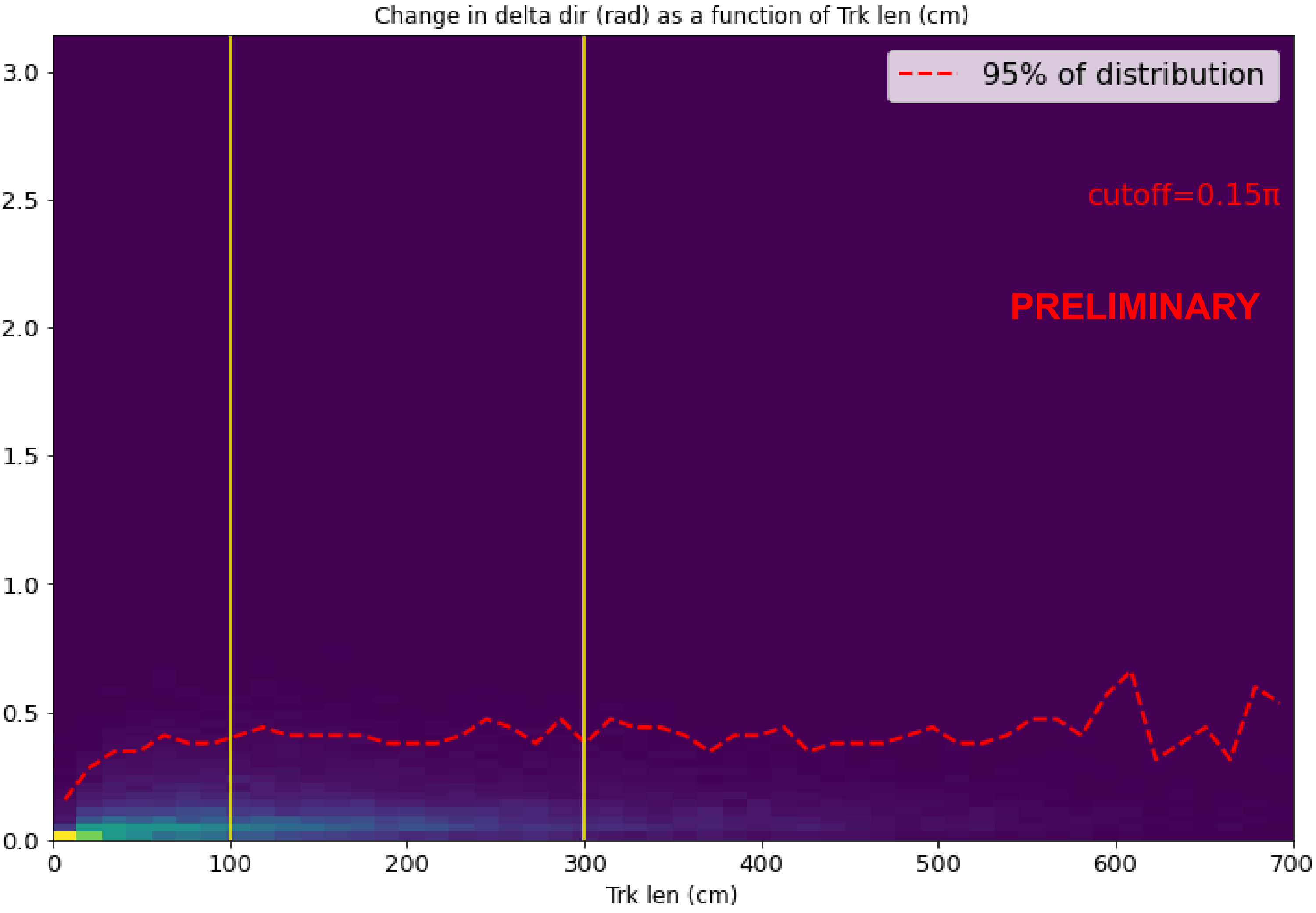
Set deviation and change in track dik len (cm) exiting tracks at track lengths equal to the expected effective track
ranges in the inactive volume (accounting for material radiation leng

Momentum From Range on Crossing Tracks

- Tracks exiting anywhere except $|x|=72$ are cut

- We calculate an "effective range" of the particle by taking a weighted sum through each material it passes through (weighted by density)

- Range calculation is more accurate in terms of bias and SD than currently used MCS based estimates (which may improve in the future)

- Tail for momentum $\quad k_{i}=\frac{38 d \text { " } A l \text { " }+40 d_{a i r}+65.88 d_{L A r}}{143.88 d_{L A r}}$ $\begin{array}{cc}\text { from range is a matter } & 143.88 d_{L A r} \\ \text { for further investigation } & \ell_{\text {eff }}=\ell_{0}+\ell_{1}+\Delta_{\text {trk }} \cdot k_{i}\end{array}$

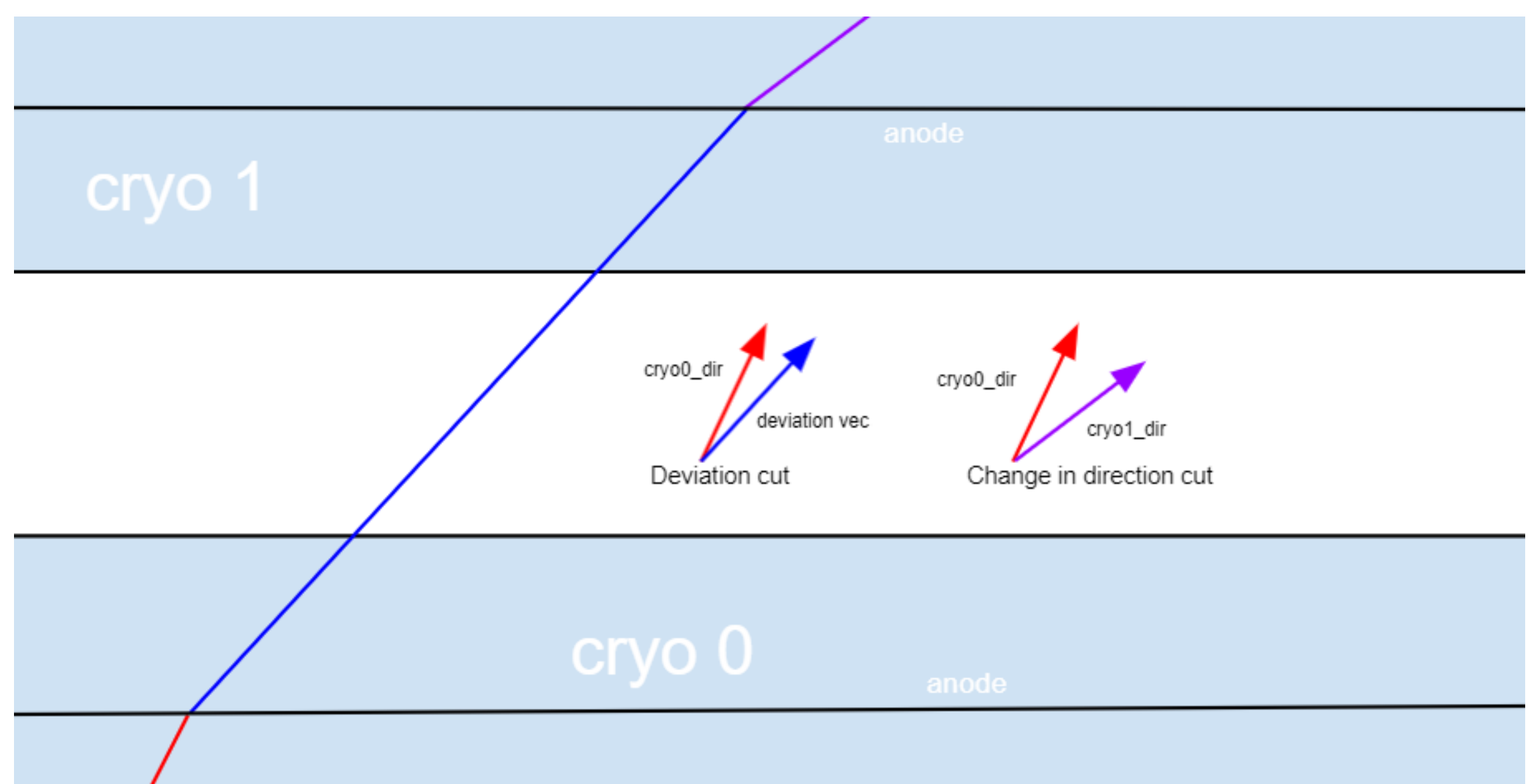

Deviation and change in track direction is the angle between the color-coded vectors (red+blue for deviation and red+purple for change in direction)

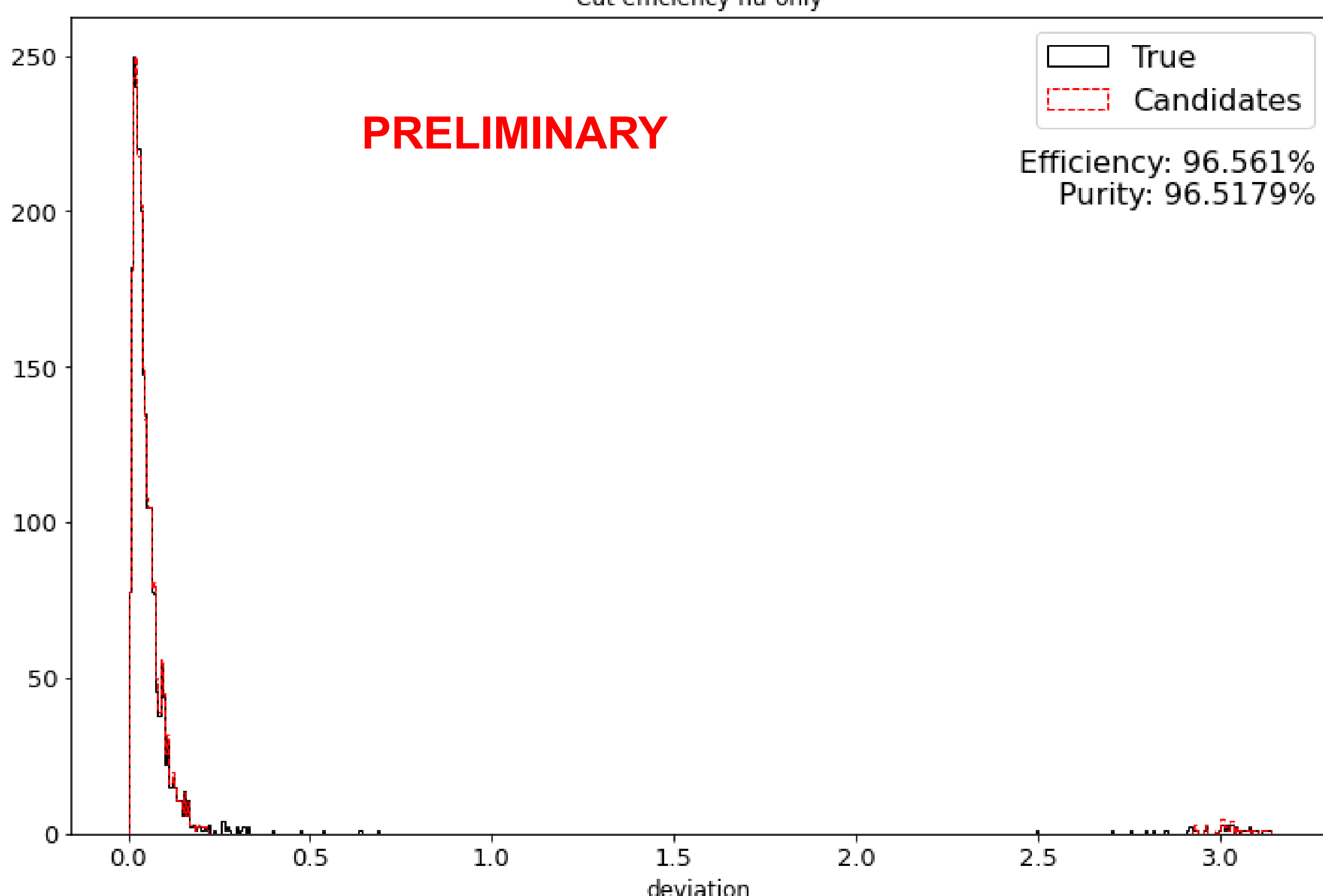

Deviation angle for true crossers and and candidate crossers. Both passing signal and purity are relatively high. True crossers are
defined as crossing particles with at least one reconstructed ack in each cryostat after cutting likely cosmics.

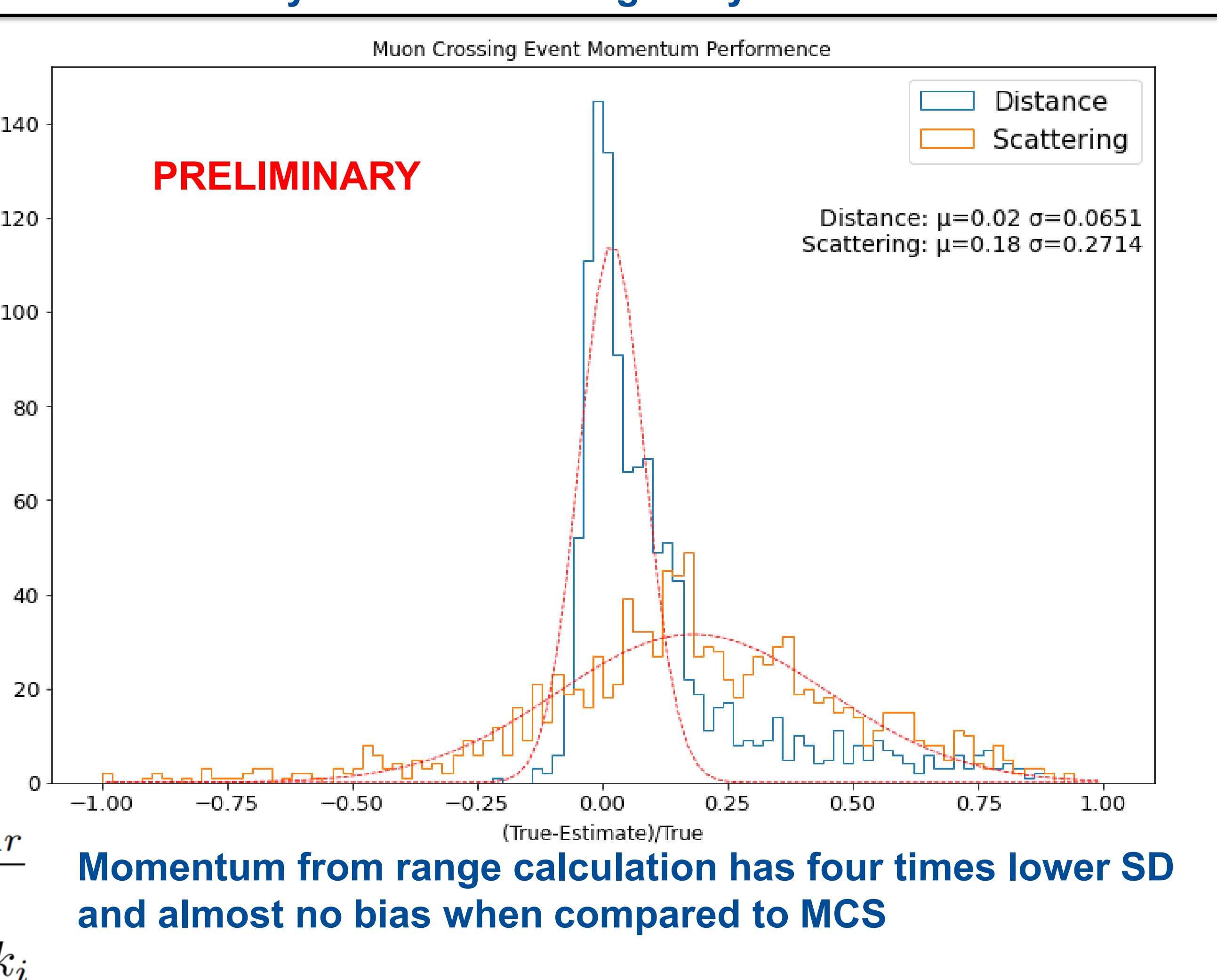
for further investigation

crossing particles with well reconstructed tracks have been well-identified in nuonly MC and show improved momentum measurements. These updated algorithms/definitions will be studied in samples with cosmics to better understand the effects of backgrounds. 